\title{
An HC-PCF Fluorescence Spectrocopy for Detection of Microsphere Samples Based on Refractive Index Scaling Law
}

\author{
Vengalathunadakal K. Shinoj ${ }^{1}$, Vadakke. M. Murukeshan ${ }^{1}$ \\ ${ }^{1}$ School of Mechanical and Aerospace Engineering, Nanyang Technological University, Singapore. \\ E-mail:mmurukeshan@ntu.edu.sg \\ Received April 13, 2011; revised May 15, 2011; accepted May 23, 2011
}

\begin{abstract}
This paper illustrates an efficient fluorescence detection of micro particles using hollow-core photonic crystal fibers (HC-PCFs) by applying the refractive index (RI) scaling law. The variations in the central wavelength for different filling material indices are illustrated for most commonly available HC-PCFs that have cladding made of pure fused silica with array of air holes running along the entire length of the fiber. The proposed concept is verified by immobilizing fluorescent microsphere samples inside two HC-PCFs of different central wavelengths and the quantification of fluorescence inside the fibers is performed through spectroscopic analysis. The sensitivity has been compared for similar fiber with different dispersed media and different fibers with same dispersed medium.
\end{abstract}

Keywords: Optical Fiber, Hollow-Core Photonic Crystal Fiber, Refractive Index Scaling, Fluorescence Spectroscopy

\section{Introduction}

It is reported that the detection sensitivity of small amount of biological threats can be enhanced with simple inexpensive methods which can give brightest possible fluorescence for detection using high throughput suspension arrays [1,2]. Moreover, many biomolecules are not available in large quantities which limit the usage of reagents in such a small environment. It is therefore important to develop optical elements or systems which can be made efficient and potent in small quantities and can be used repeatedly. The use of optical fibers for various sensing purposes has been reported $[3,4]$. The emergence of microstructured optical fibers (MOFs) opens up new opportunities for novel fluorescent detection and relevant biosensor design, which can solve the problems encountered in conventional biosensors [5-7]. MOFs are characterized as having a plurality of air holes running along the entire length of the fiber [8]. The optical properties of this class of fibers are determined by their geometry, size, and relative position of the air holes. Photonic crystal fibers (PCFs) are one of the most prominent MOFs that have emerged in recent years that could be engineered to have vastly different properties compared to conventional fibers $[9,10]$. Its guiding mechanism is based on the photonic bandgap formed due to its high index contrast (commonly silica and air in optical region) and from the wavelength-scale microstructure. The mode propagation properties strongly depend on wavelength, which in turn depends on the design, configuration and geometry of air holes [11]. Unlike conventional fibers, photonic crystal fibers are made of pure silica glass $\left(\mathrm{SiO}_{2}\right)$ without any doping. Hence it is biocompatible and chemically inert [12]. Further, the capillary tubes present in the PCFs have a good surfaceto-volume ratio. The PCF-based sensor hence utilizes the available sample volume much more efficiently.

The hollow-core PCFs (HC-PCFs) are comprised of an air core with a cladding that consists of a two-dimensional (2-D) periodic array of air inclusions in silica [13]. As indicated by their name, HC-PCFs guide light in the air core within certain bandgaps, which manifest as transmission windows in the transmission spectrum. The photonic bandgap (PBG) property of the fiber is a function of both its geometry and the refractive-index (RI) contrast $[10,13]$. The transmission bands, or transmission windows, of the HC-PCF is decided by the spacing between the holes of the capillaries (pitch), the hole diameters of the capillaries, and/or the air-filling content within the inner cladding. When the holey regions of HC-PCFs are filled with aqueous solution, the transmission window shows a blue shift [14-18]. This approach is in analogous with the wellknown scaling laws that describe the shift in the PBG edge 
which is derived from scalar waveguide approximation $[14,15]$. By means of the scalar-wave approximation, simple index RI scaling laws have been derived to predict the manner in which the photonic states of the fiber scale with changes in the refractive-index contrast [14]. An experimental demonstration of the shift in the PBG edge due to refractive-index scaling using $\mathrm{D}_{2} \mathrm{O}$-filled $\mathrm{HC}$-PCFs has reported based on the above approximation [16]. The application of HC-PCF as a refractive index sensor based on RI scaling laws has also been reported [17]. Recently, the dependence of PBG edge shift on the physical measurands such as strain, temperature, curvature, and twist are studied [18]. In this context, this paper investigates the influence of shift in central wavelength on the fluorescence emission intensity in common fluorescence sensing studies employing HC-PCFs.

\section{Theoretical Background}

In Hollow-core Photonic crystal fibers (HC-PCFs) are characterized as having a hollow-core surrounded by pattern of air holes running along the entire length of the fiber. Filling the holes of such a fiber with liquid will change the refractive index of the holey region and therefore will result in the shift of band gaps and their operational bandwidths. The shift in bandgap can be estimated by refractive index scaling law which is derived from scalar waveguide approximation $[14,16]$.

$$
\lambda=\lambda_{0}\left[\left(n_{b}^{2}-n_{m}^{2}\right) /\left(n_{b}^{2}-n_{a}^{2}\right)\right]^{1 / 2}
$$

In Equation (1), ' $n_{a}$ ' represents the ambient index inside the holey region, which includes the core and the holes inside the cladding. The refractive index of background material and infiltrated material is denoted as ' $n_{b}$ ' and ' $n_{m}$ ' respectively. Also, $\lambda_{0}$ represents the central wavelength of the fiber in air medium $\left(n_{0}\right)$. Hence for hollow-core fibers with similar geometry profile, when the refractive index of the filling material changes from $n_{0}$ to $n_{m}$, the corresponding wavelength shift of the PBG edge varies from $\lambda_{0}$ to $\lambda$. Differentiating Equation (1),

$$
\begin{aligned}
\frac{\mathrm{d} \lambda}{\mathrm{d} n_{m}} & =-\frac{\lambda_{0} \cdot n_{m}}{\left[\left(n_{b}^{2}-n_{a}^{2}\right)^{1 / 2} \cdot\left(n_{b}^{2}-n_{m}^{2}\right)^{1 / 2}\right]} \\
\frac{\mathrm{d} \lambda}{\mathrm{d} n_{m}} & =-K \cdot \frac{n_{m}}{\left(n_{b}^{2}-n_{m}^{2}\right)^{1 / 2}}
\end{aligned}
$$

where,

$$
K=\frac{\lambda_{0}}{\left(n_{b}^{2}-n_{a}^{2}\right)^{1 / 2}}
$$

' $K$ ' is a positive constant determined by the refractive index of the fiber material and the central wavelength. $\left(\mathrm{d} \lambda / \mathrm{d} n_{m}\right)$ represents the refractive index sensitivity and its negative value indicates that the PBG has a blue-shift in wavelength with increase in index of the infiltrated material. $\left(\mathrm{d} \lambda / \mathrm{d} n_{m}\right)$ varies with the ambient refractive index $\left(n_{a}\right)$ of the medium, background material index $\left(n_{b}\right)$ and infiltrated material index $\left(n_{m}\right)$.

Most HC-PCFs have cladding made of pure fused silica $\left(n_{b}=1.45\right)$ with array of air holes $\left(n_{a}=1\right)$ running along the entire length of the fiber. In HC-PCF based fluorescence sensing applications, the sample volume is drawn into the fiber holes using capillary action. In general, the fluorescence samples are dissolved/dispersed in medium such as methanol, water or ethanol etc. Evaluation of Equation (1) and Equation (3) for different filling material indices ranging from 1.3 to 1.4 are performed for two hollow-core fibers (from Crystal Fiber A/S) with central wavelengths $830 \mathrm{~nm}$ (HC-800-01) and $1060 \mathrm{~nm}$ (HC-1060-02) which are employed in the experimental study (section 3). The obtained results given in Figure 1(a) and Figure 1(b) denote the variation in the central wavelength and refractive index sensitivity, respectively, for different filling liquid indices. Based on the RI scaling law (Equation (1)), for a particular filling material, the shifted wavelength $(\lambda)$ is proportional to central wavelength $\left(\lambda_{0}\right)$ of the HC-PCF. The variation of $\lambda$ with $\lambda_{0}$ is plotted for different filling material indices values ranging from 1.3 to 1.4 in Figure 2. Here also we considered most commonly available HC-PCFs that have cladding made of pure fused silica with array of air holes running along the entire length of the fiber. It can be seen that on increasing the filling material indices, the central wavelength of a particular HC-PCF is shifted to the lower wavelength region. This shift in central wavelength should be a significant parameter to be considered in $\mathrm{HC}-\mathrm{PCF}$ based fluorescent sensors where fluorescent sample solutions are infiltrated into the fiber holes. An experiment has been performed to demonstrate the induced changes in the sensitivity of the HC-PCF based fluorescence sensors due to the shift in wavelength and is explained in the following section.

\section{Experimental Study}

\subsection{Materials and Methods}

Two hollow-core fibers, HC-800-01 and HC-1060-02 are selected for the experimental study. The scanning electron micrograph (SEM) images of the HC-PCF facets are given in Figure 3(a) and Figure 3(b). The HC-800-01 has an approximate core diameter of $9.3 \mu \mathrm{m}$ surrounded by a 40 $\mu \mathrm{m}$-diameter microstructured cladding. It operates at a 

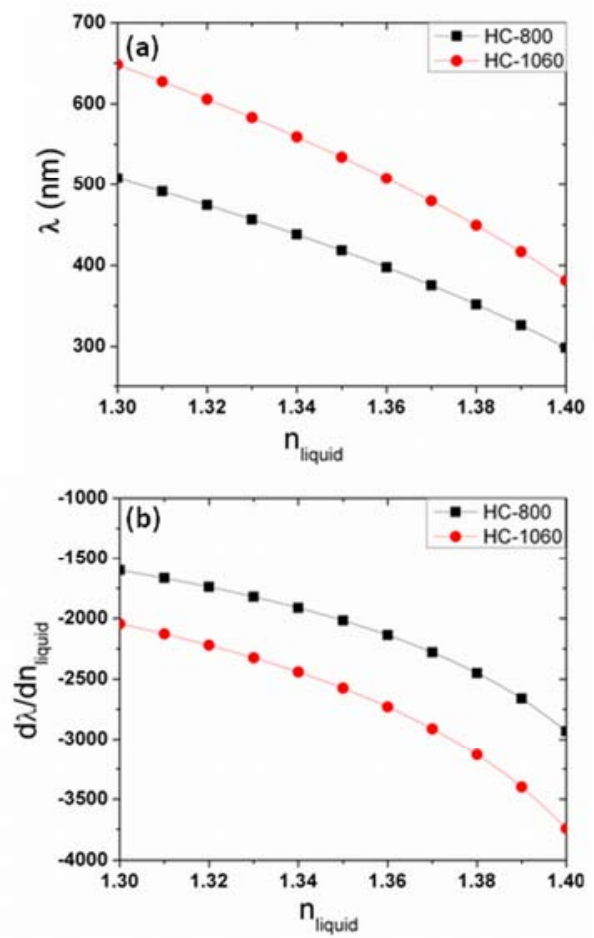

Figure 1. (a) Central wavelength plot for HC-1060 (solid circles) and HC-800 (solid rectangles) using Equation (1) and (b) refractive index sensitivity plot for $\mathrm{HC}-1060$ (solid circles) and HC-800 (solid rectangles) using Equation (3), for different filling indices between 1.3 and 1.4.

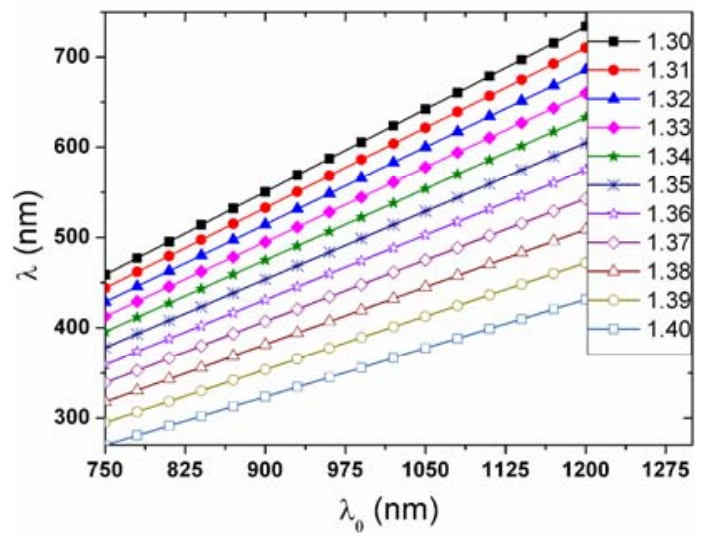

Figure 2. The shift in central wavelength $\left(\lambda_{0}\right)$ of HC-PCFs to the new wavelength $(\lambda)$ at various filling material indices.

center wavelength of $830 \mathrm{~nm}$ and exhibits full photonic bandgap (high transmission range) extending from approximately $770 \mathrm{~nm}$ to $890 \mathrm{~nm}$. The attenuation over this range is less than $0.5 \mathrm{~dB} / \mathrm{m}$. While HC-1060-02 hollowcore photonic bandgap presents a band larger than 100 $\mathrm{nm}$ centred at $1060 \mathrm{~nm}$. The hollow core has a centre core size of diameter $10 \pm 1 \mu \mathrm{m}$ surrounded by a microstructure comprised of eight periods of hexagonally



Figure 3. SEM images of HC-PCF with (a), central wavelength $830 \mathrm{~nm}(\mathrm{HC}-800) \&(b)$ central wavelength $1060 \mathrm{~nm}$ (HC-1060) and microscopic Side view of (c) cleaved HC-800 end (imaged with10X/0.3NA objective lens) and (d) green fluorescent microspheres, of size $2 \mu \mathrm{m}$, immobilized inside the HC- PCF (imaged with 50X/0.75NA Objective lens).

packed cylinders with a period of $2.75 \mu \mathrm{m}$ and a filling fraction of around $90 \%$. The cladding diameter is $123 \pm 5$ $\mu \mathrm{m}$. Both the hollow-core fibers are cut into segments of $\approx 10 \mathrm{~cm}$ length and one end of the fiber is cleaved carefully using a fiber cleaver to produce a flat surface. Microscopic side view of the cleaved fiber end is given in Figure 3(c).

The green fluorescent microspheres (Duke Scientific Corp.), of diameter $\approx 2 \mu \mathrm{m}$, employed in this study are internally-dyed polymer beads. The particles are in a solution of DI water and some surfactants. The green fluorescence labeled microsphere immobilized fiber that gives an emission maximum wavelength at around $508 \mathrm{~nm}$ is excited with blue laser light $(473 \mathrm{~nm})$. In order to verify the influence of photonic bandgap edge shift on the sensitivity of fluorescence signal, two types of study has been performed. In the first study, same fiber (with central wavelength $830 \mathrm{~nm}$ ) has been used for two different dispersion media such as ethanol $(n=1.36)$ and distilled water $(n=$ 1.33). In the second case, two fibers with different central wavelengths $(830 \mathrm{~nm}$ and $1060 \mathrm{~nm})$ are considered with sample particles are dispersed in same medium (ethanol). The experiment is carried out on both fibers for same values of laser power and similar coupling efficiency in order to compare the fluorescence collection efficiency.

The cleaved end of the HCPCFs segments were dipped into the sample solution to allow the sample to drawn into the fiber due to the capillary effect. The microsphere particles had nearly the same density as water $\left(1.05 \mathrm{~g} / \mathrm{cm}^{3}\right)$. Therefore, the particles would follow the fluid flow arising from the capillary force. The presence of the microsphere sample inside the fiber is detected using a fluorescence microscope. The obtained fluores- 
cent microscopic picture of fiber containing fluorescent microspheres is shown Figure 3(d). The quantification of fluorescence signal from both fibers is performed using spectroscopic analysis as described below.

\subsection{Spectroscopic Analysis}

Schematic diagram of the experimental setup is shown in Figure 4. A continuous wave (CW) diode-pumped solidstate (DPSS) $473 \mathrm{~nm}$ laser (output power $10 \mathrm{~mW}$ ) is coupled into the proximal end of PCF, immobilized with fluorescence sample, using a high precision single mode fiber coupling (FC) unit (Melles Griot Pte Ltd) with a microscope objective $\left(20 \mathrm{X}, 0.65 \mathrm{NA}\left(\mathrm{L}_{1}\right)\right.$. The diverging light beam emerging from the distal end of the sample immobilized fiber is collimated using a microscope objective lens [Newport M-20X/0.4 $\left.\left(\mathrm{L}_{2}\right)\right]$. The parallel beam emerging from this objective lens is focused onto the entrance slit of the high quantum efficiency spectrometer using another microscope objective lens [Newport $\left.\mathrm{M}-40 \mathrm{X} / 0.65\left(\mathrm{~L}_{3}\right)\right]$. The spectrometer is coupled to a $\mathrm{PC}$ which displays the spectrum.

The fluorescent spectra obtained at identical conditions from HC-800 fiber segments for ethanol and water dispersed microsphere samples are normalized as shown in Figure 5. The water dispersed fiber gives better signal for green fluorescence when compared to the ethanol dispersed fiber. This result is in agreement with the result obtained in section 2 (Figure 1 and Figure 2). It can be inferred from Figure 2 that for a fiber with central wavelength $\left(\lambda_{0}\right) 830 \mathrm{~nm}$ (HC-800), the filling of water causes the shift in central wavelength to an approximate value of $457 \mathrm{~nm}$. But, the filling of ethanol shifts the central wavelength from $830 \mathrm{~nm}$ to $397 \mathrm{~nm}$, approximately. Hence the water filled HC-800 has central wavelength nearer to the green region which results in better sensitivity. The results are found to be reproducible for different fiber segments with same central wavelength $(830 \mathrm{~nm})$.

Figure 6 shows the normalized spectra obtained from fibers with central wavelengths $830 \mathrm{~nm}$ and $1060 \mathrm{~nm}$ for green fluorescent particles dispersed in ethanol medium. It is vivid that the fiber with central wavelength $1060 \mathrm{~nm}$

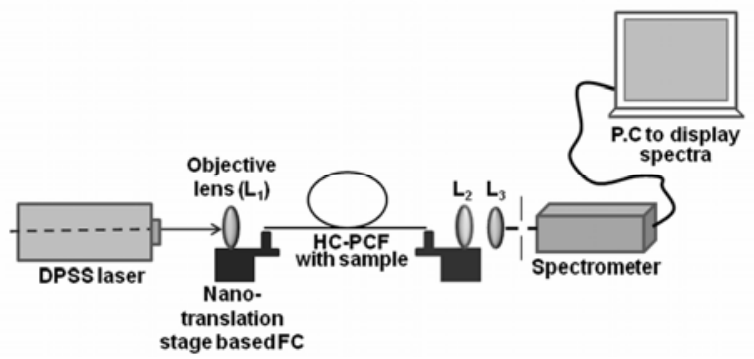

Figure 4. Schematic diagram-experimental set up used for the spectral analysis.

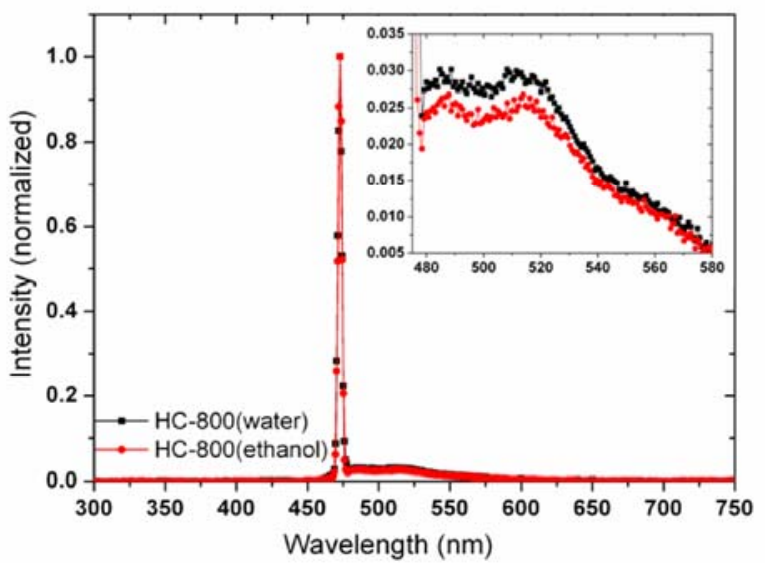

Figure 5. The obtained fluorescence spectrum (normalized) at $473 \mathrm{~nm}$ excitation from the HC-PCF with central wavelength $830 \mathrm{~nm}$ filled with green fluorescent microparticles dispersed in water (black solid rectangles) and ethanol (red solid circles) [Inset: the wavelength region corresponding to the fluorescence emission is expanded].

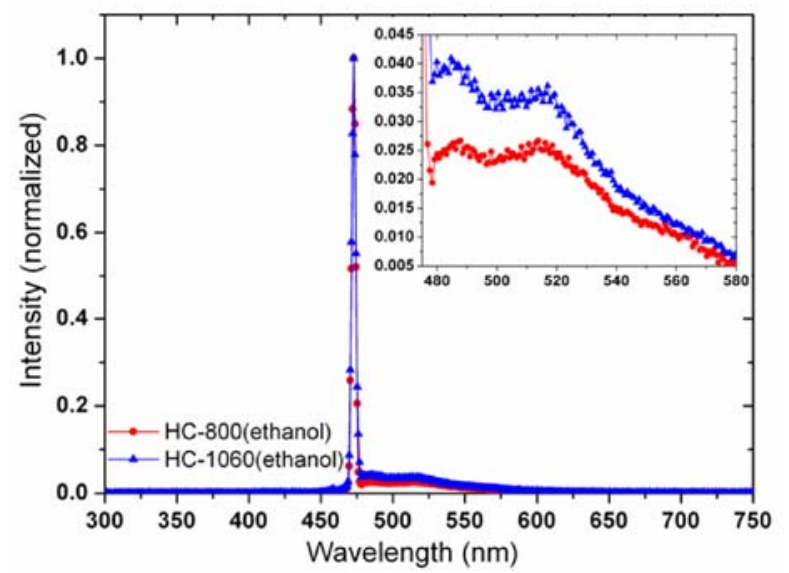

Figure 6. The obtained fluorescence spectrum (normalized) at $473 \mathrm{~nm}$ excitation from the HC-PCFs with central wavelengths $830 \mathrm{~nm}$ (red solid circles) and $1060 \mathrm{~nm}$ (blue solid triangles) filled with green fluorescent microparticles dispersed in ethanol [Inset: the wavelength region corresponding to the fluorescence emission is expanded].

shows better fluorescent signal when compared with fiber of central wavelength $830 \mathrm{~nm}$. The higher intensity obtained with $1060 \mathrm{~nm}$ fiber is also in agreement with the results shown in section 2 , corresponding to refractive index 1.36 which is refractive index of the dispersed medium (ethanol). From Figure 2, it can be seen that for a filling material index of 1.36 , central wavelength of the HC-1060 fiber will shift from $1060 \mathrm{~nm}$ to around $508 \mathrm{~nm}$. Whereas in the case of fiber with central wavelength at $830 \mathrm{~nm}$ (HC-800), the approximate value of shifted wavelength is $400 \mathrm{~nm}$ for the infiltrated material index of 


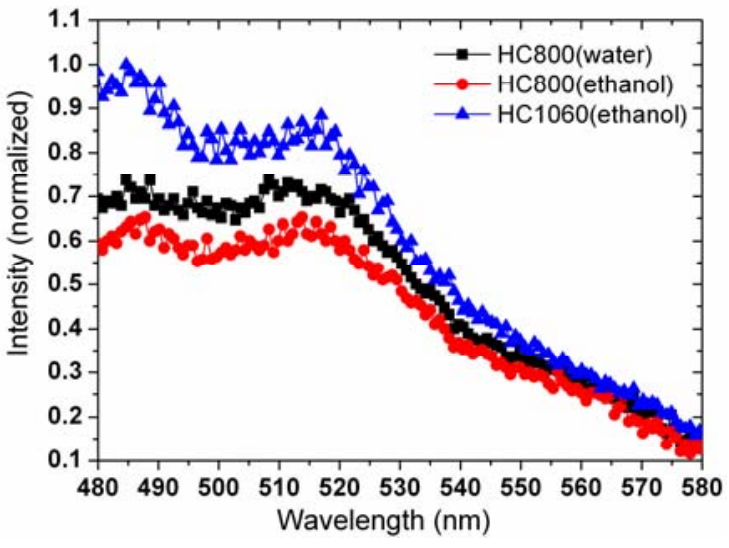

Figure 7. Comparison of green fluorescent signals obtained from the $\mathrm{HC}-800$ filled with green fluorescent microparticles dispersed in water (black solid rectangles) and ethanol (red solid circles), and HC-1060 filled with green fluorescent microparticles dispersed in ethanol (blue solid triangles).

1.36. The results are repeatable for different fiber segments with the same central wavelengths. The fluorescent spectroscopic signals obtained (which are shown in Figure 5 and Figure 6 are plotted in Figure 7 for intensity comparison. The obtained results are found to be in accordance with the simulation done based on RI scaling law.

\section{Conclusions}

In conclusion, an $\mathrm{HC}-\mathrm{PCF}$ fluorescence spectroscopic scheme has been illustrated on the basis of refractive index scaling law. The variations in the central wavelength for different filling material indices are analyzed in the case of HC-PCFs with cladding made of pure fused silica with array of air holes running along the entire length of the fiber. A proof of concept study has been performed by infiltrating fluorescence sample volume inside HC-PCF and the quantification of fluorescence intensity is analyzed using spectroscopic method. The sensitivity has been compared for similar fiber with different dispersed media and different fibers with same dispersed medium. The obtained experimental results are in good agreement with the analytical simulation results. These findings are expected to accelerate the R\&D on HC-PCF based ultrasensitive spectroscopic analysis and relevant sensors for specific detection of biomolecules in very low sample volumes.

\section{Acknowledgements}

The authors acknowledge the financial support received through ASTAR-SERC and ARC (MOE). One of the authors, V. K. Shinoj, would also like to acknowledge Nanyang Technological University for the research stu- dent support.

\section{References}

[1] E. Brooks Shera, N. Seitzinger, L. Davis, R. Keller and S. Soper, "Detection of single fluorescent molecules," Chemical Physics Letters, Vol. 174, No. 6, 1990, pp. 553-557. doi:10.1016/0009-2614(90)85485-U

[2] A. Castro, and E. Shera, "Single-Molecule Detection: Applications to Ultrasensitive Biochemical Analysis," Applied Optics, Vol. 34, No. 18, 1995, pp. 3218-3222. doi:10.1364/AO.34.003218

[3] Y. Zhao, and Y. Liao, "Discrimination Methods and Demodulation Techniques for Fiber Bragg Grating Sensors," Optics and Lasers in Engineering, Vol. 41, No. 1, 2004, pp. 1-18. doi:10.1016/S0143-8166(02)00117-3

[4] J. I. Peterson, and G. G. Vurek, "Fiber-Optic Sensors for Biomedical Applications," Science, Vol. 224, No. 4645, 1984, pp. 123-127. doi:10.1126/science.6422554

[5] J. Jensen, P. Hoiby, G. Emiliyanov, O. Bang, L. Pedersen, and A. Bjarklev, "Selective Detection of Antibodies in Microstructured Polymer Optical Fibers," Optics Express, Vol. 13, No. 15, 2005, pp. 5883-5889. doi:10.1364/OPEX.13.005883

[6] C. Cordeiro, M. Franco, G. Chesini, E. Barretto, R. Lwin, C. Brito Cruz and M. Large, "Microstructured-Core Optical Fibre for Evanescent Sensing Applications," Optics Express, Vol. 14, No. 26, 2006, pp. 13056-13066. doi:10.1364/OE.14.013056

[7] A. S. Webb, F. Poletti, D. J. Richardson and J. K. Sahu, "Suspended-Core Holey Fiber for Evanescent-Field Sensing," Optical Engineering, Vol. 46, No. 1, 2007, p. 010503. doi:10.1117/1.2430505

[8] M. van Eijkelenborg, M. Large, A. Argyros, J. Zagari, S. Manos, N. Issa, I. Bassett, S. Fleming, R. McPhedran and C. de Sterke, "Microstructured Polymer Optical Fibre," Optics Express, Vol. 9, No. 7, 2001, pp. 319-327. doi:10.1364/OE.9.000319

[9] P. Russell, "Review: Photonic Crystal Fibers," Science, Vol. 299, 2003, pp. 358-362. doi: $10.1126 /$ science. 1079280

[10] J. C. Knight, J. Broeng, T. A. Birks and P. S. J. Russell, "Photonic Band Gap Guidance in Optical Fibers," Science, Vol. 282, No. 5393, 1998, pp. 1476-1478. doi: $10.1126 /$ science. 282.5393 .1476

[11] D. Mogilevtsev, T. A. Birks and P. S. J. Russell, "Group-Velocity Dispersion in Photonic Crystal Fibers," Optics Letters, Vol. 23, No. 21, 1998, pp. 1662-1664. doi:10.1364/OL.23.001662

[12] S. Padmanabhan, V. K. Shinoj, V. M. Murukeshan and P. Padmanabhan, "Highly Sensitive Optical Detection of Specific Protein in Breast Cancer Cells Using Microstructured Fiber in Extremely Low Sample Volume," Journal of Biomedical Optics, Vol. 15, No. 1, 2010, pp. 017005-017006. doi:10.1117/1.3302810

[13] R. F. Cregan, B. J. Mangan, J. C. Knight, T. A. Birks, P. S. J. Russell, P. J. Roberts and D. C. Allan, "Single-Mode 
Photonic Band Gap Guidance of Light in Air," Science Vol. 285, No. 5344, 1999, 1537. doi:10.1126/science.285.5433.1537

[14] T. Birks, D. Bird, T. Hedley, J. Pottage and P. Russell, "Scaling Laws and Vector Effects in Bandgap-Guiding Fibres," Optics Express, Vol. 12, No. 1, 2004, pp. 69-74. doi:10.1364/OPEX.12.000069

[15] J. Joannopoulos, R. Meade and J. Winn, "Photonic Crystals," Princeton University Press, Princeton, 1995.

[16] G. Antonopoulos, F. Benabid, T. A. Birks, D. M. Bird, J. C. Knight and P. S. J. Russell, "Experimental Demonstration of the Frequency Shift of Bandgaps in Photonic Crystal Fibers Due to Refractive Index Scaling," Optics
Express, Vol. 14, No. 7, 2006, pp. 3000-3006. doi:10.1364/OE.14.003000

[17] J. Sun and C. C. Chan, "Photonic bandgap fiber for refractive index measurement," Sensors and Actuators B: Chemical, Vol. 128, No. 1, 2007, pp. 46-50. doi:10.1016/j.snb.2007.05.037

[18] S. H. Aref, R. Amezcua-Correac, J. P. Carvalho, O. Frazão, J. L. Santos, F. M. Araújo, H. Latifi, F. Farahi, L. A. Ferreira and J. C. Knight, "Spectral Characterization of a Photonic Bandgap Fiber for Sensing Applications," Applied Optics, Vol. 49, No. 10, 2010, pp. 1870-1875 doi:10.1364/AO.49.001870 